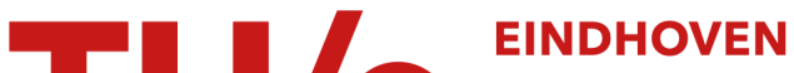 \\ UNIVERSITY OF \\ TECHNOLOGY
}

\section{Rheology across the zero-temperature jamming transition}

\section{Citation for published version (APA):}

Paredes Rojas, J., Michels, M. A. J., \& Bonn, D. (2013). Rheology across the zero-temperature jamming transition. Physical Review Letters, 111(1), 1-5. [015701]. https://doi.org/10.1103/PhysRevLett.111.015701

DOI:

10.1103/PhysRevLett.111.015701

Document status and date:

Published: 01/01/2013

\section{Document Version:}

Publisher's PDF, also known as Version of Record (includes final page, issue and volume numbers)

\section{Please check the document version of this publication:}

- A submitted manuscript is the version of the article upon submission and before peer-review. There can be important differences between the submitted version and the official published version of record. People interested in the research are advised to contact the author for the final version of the publication, or visit the $\mathrm{DOI}$ to the publisher's website.

- The final author version and the galley proof are versions of the publication after peer review.

- The final published version features the final layout of the paper including the volume, issue and page numbers.

Link to publication

\section{General rights}

Copyright and moral rights for the publications made accessible in the public portal are retained by the authors and/or other copyright owners and it is a condition of accessing publications that users recognise and abide by the legal requirements associated with these rights.

- Users may download and print one copy of any publication from the public portal for the purpose of private study or research.

- You may not further distribute the material or use it for any profit-making activity or commercial gain

- You may freely distribute the URL identifying the publication in the public portal.

If the publication is distributed under the terms of Article 25fa of the Dutch Copyright Act, indicated by the "Taverne" license above, please follow below link for the End User Agreement:

www.tue.nl/taverne

Take down policy

If you believe that this document breaches copyright please contact us at:

openaccess@tue.nl

providing details and we will investigate your claim. 


\title{
Rheology across the Zero-Temperature Jamming Transition
}

\author{
José Paredes, ${ }^{1}$ Matthias A. J. Michels, ${ }^{2}$ and Daniel Bonn ${ }^{1}$ \\ ${ }^{1}$ Van der Waals-Zeeman Institute, Institute of Physics, University of Amsterdam, Science Park 904, \\ 1098 XH Amsterdam, The Netherlands \\ ${ }^{2}$ Department of Applied Physics, Theory of Polymers and Soft Matter, Eindhoven University of Technology, \\ P.O. Box 513, 5600 MB Eindhoven, The Netherlands
}

(Received 9 March 2013; published 2 July 2013)

\begin{abstract}
Many soft-matter systems show a transition between fluidlike and mechanically solidlike states when the volume fraction of the material, e.g., particles, drops, or bubbles is increased. Using an emulsion as a model system with a precisely controllable volume fraction, we show that the entire mechanical behavior in the vicinity of the jamming point can be understood if the mechanical transition is assumed to be analogous to a phase transition. We find power-law scalings in the distance to the jamming point, in which the parameters and exponents connect the behavior above and below jamming. We propose a simple twostate model with heterogeneous dynamics to describe the transition between jammed and mobile states. The model reproduces the steady-state and creep rheology and relates the power-law exponents to diverging microscopic time scales.
\end{abstract}

DOI: 10.1103/PhysRevLett.111.015701

PACS numbers: 64.60.- i, 47.50.- d, 47.57.Bc, 83.50.-v

Understanding and ultimately predicting the flow behavior of complex fluids is a subject of considerable fundamental and practical importance [1]. Most frequently, complex fluids are dispersions of one material in a continuous phase, e.g., suspensions of particles or polymers, foams, and emulsions. The common denominators in the mechanical behavior are the emergence of a yield stress for volume fractions higher than some critical value $\phi_{c}$ and Newtonian flow for lower volume fractions, with shear thinning in either case for high shear rates. This generic behavior is mostly described by empirical equations. For concentrated systems with $\phi>\phi_{c}$, flow curves are often successfully described by the phenomenological HerschelBulkley equation [2], $\sigma=\sigma_{y}+K \dot{\gamma}^{\beta}$, where $\sigma$ is the stress, $\sigma_{y}$ is the yield stress, $\dot{\gamma}$ is the shear rate, and $K$ and $\beta$ are adjustable model parameters. For $\phi<\phi_{c}$ the flow behavior is usually described by a Cross-type equation [3], $\sigma=\eta_{1} \dot{\gamma} /\left(1+C \dot{\gamma}^{1-\delta}\right)$, where $\eta_{1}$ is the low-shear viscosity, and $C$ and $\delta$ are again adjustable parameters. Additionally, it is customary to describe the influence of the volume fraction on the low-shear viscosity using the-again phenomenological-Krieger-Dougherty equation [4], $\eta_{1}=\eta_{s}\left(1-\phi / \phi_{c}\right)^{-m}$, where we identify the low-shear viscosity of the Cross equation as $\eta_{1}, \eta_{s}$ is the viscosity of the suspending medium, and $m$ is a free-fitting parameter with a value $1.5 \lesssim m \lesssim 2$ [5]. Thus, all these phenomenological expressions employ a variety of exponents and amplitudes to describe the flow behavior.

Especially for concentrated suspensions, it has proven very difficult to deal with the long-range hydrodynamic interactions and derive such behavior from first principles [1]. This is why in recent years a generic framework has been proposed for concentrated complex fluids based on jamming, which denotes the transition between a flowing and a stagnant state $[6,7]$. A material is said to be jammed when for $\phi>\phi_{c}$ the system will not flow unless $\sigma_{y}$ is overcome. Only a few models, simulations, and experiments have been proposed or carried out for describing how flow properties of such systems behave with respect to $\phi$ or the distance to $\phi_{c}(|\Delta \phi|)$ [7-11]. These models depend on the interaction potential between the dispersed particles, and some of them lead to master curves when flow curves are rescaled with respect to $\Delta \phi[8-10,12]$; however to which degree the rescaling and corresponding exponents are universal is not yet clear. A recent simulation study [13], considering also the interaction potentials, was able to give a unified picture of glass and jamming rheology; in particular, glassy arrest of thermal systems and athermal jamming were shown to be unrelated. In [13], the athermal jamming transition is found at $\phi_{c} \approx 0.64$, well above the glass transition, as we find here.

The central question we ask in this Letter is whether the "zero-temperature jamming transition" for athermal particles can be understood in a way that is analogous to a usual phase transition, which happens here as a function of $\phi$. If this could be done, the behaviors above and below $\phi_{c}$ should be connected, and consequently a measurement of the flow curve above $\phi_{c}$ would give predictions on the behavior in the unjammed state and vice versa. We consider jamming in a simple material made of soft repulsive particles that exhibits a jamming transition. Such repulsive systems are the best model systems since interparticle attractions induce thixotropy, which makes the yield stress ill defined $[14,15]$.

We use an emulsion as our model system to show how the rheological properties at $\phi>\phi_{c}$ and $\phi<\phi_{c}$ scale with respect to $|\Delta \phi|$, the distance to jamming. These data show that the scalings and the corresponding scaling 
exponents are interconnected, and that the values above and below jamming can be interrelated experimentally. Subsequently we propose a simple structure-based model, with the aim of understanding the transition across $\phi_{c}$ from a microscopic picture of heterogeneous dynamics.

Our experimental system is an emulsion of castor oil in water, stabilized using sodium dodecyl sulfate (SDS) [14]. A batch of emulsion with $\phi \approx 0.8$ (mean drop diameter $=3.2 \mu \mathrm{m}$ and $20 \%$ polydispersity) is prepared by dispersing the oil in a 1 wt $\%$ SDS solution; at this surfactant concentration, emulsions have a yield stress that is due only to repulsive forces between the droplets [14-16]. From this batch, samples with lower $\phi$ are obtained by dilution with the 1 wt $\%$ SDS solution. Rheological measurements are performed using the following three rheometers, two of which have a coneplate geometry (with roughened surfaces) [17]: (i) a controlled-shear-rate rheometer [Rheometrics ARES], (ii) a controlled-shear-stress rheometer (CSS) [Anton Paar MCR 300]; and (iii) another controlled-shear-stress rheometer (CSS) [Stresstech] with a vane-in-cup geometry and roughened cup. Before carrying out any experiment, samples are presheared at $100 \mathrm{~s}^{-1}$ for $30 \mathrm{~s}$ and left to relax for $30 \mathrm{~s}$ in order to assure the same initial condition for all measurements.

A first experiment consists in obtaining flow curves of all the samples using the controlled-shear-rate rheometer, by performing shear-rate sweeps as shown in Fig. 1(a). From these flow curves we determined that $\phi_{c} \approx 0.645$ as linear extrapolation of the yield stress to zero gives $\phi_{c}=$ $0.648 \pm 0.004$, whereas a quadratic fit $\sigma_{y}=A\left(\phi-\phi_{c}\right)^{2}$ gives $\phi_{c}=0.645 \pm 0.005$. On the one hand, flow curves with $\phi>\phi_{c}$ are fitted to the Herschel-Bulkley equation. These curves show that samples with $\phi>\phi_{c}$ have a yield stress and behave like shear-thinning fluids; i.e., the exponent $\beta$ is lower than 1 . On the other hand, samples with $\phi<\phi_{c}$ do not have a yield stress; these emulsions exhibit a Newtonian-flow behavior at low shear rates, while at high shear rates they exhibit shear-thinning behavior near $\phi_{c}$. The value of $\phi_{c}$ is close to the expected value for random close packing, $\phi_{\mathrm{RCP}} \approx 0.64$.

We rescale all flow curves for different volume fractions onto a single master plot by plotting $\sigma /|\Delta \phi|^{\Delta}$ versus $\dot{\gamma} /|\Delta \phi|^{\Gamma}$, which allows us to collapse all flow data above $\phi_{c}$ by fitting $\Delta=2.13$ and $\Gamma=3.84$, see Fig. 1(b). Interestingly, this procedure automatically collapses also the flow data below $\phi_{c}$, onto a different master curve that merges with the one above $\phi_{c}$ for high shear rates. The branch corresponding to fractions above $\phi_{c}$ can be fitted to a Herschel-Bulkley equation with $\beta=\Delta / \Gamma=0.55$ and $K=0.87$. In addition, the branch corresponding to fractions below $\phi_{c}$ can be fitted to a Cross-type equation in a particular form that, by considering the Krieger-Dougherty equation, takes into account both the low-shear Newtonian regime and the shear-thinning regime at high shear rates,
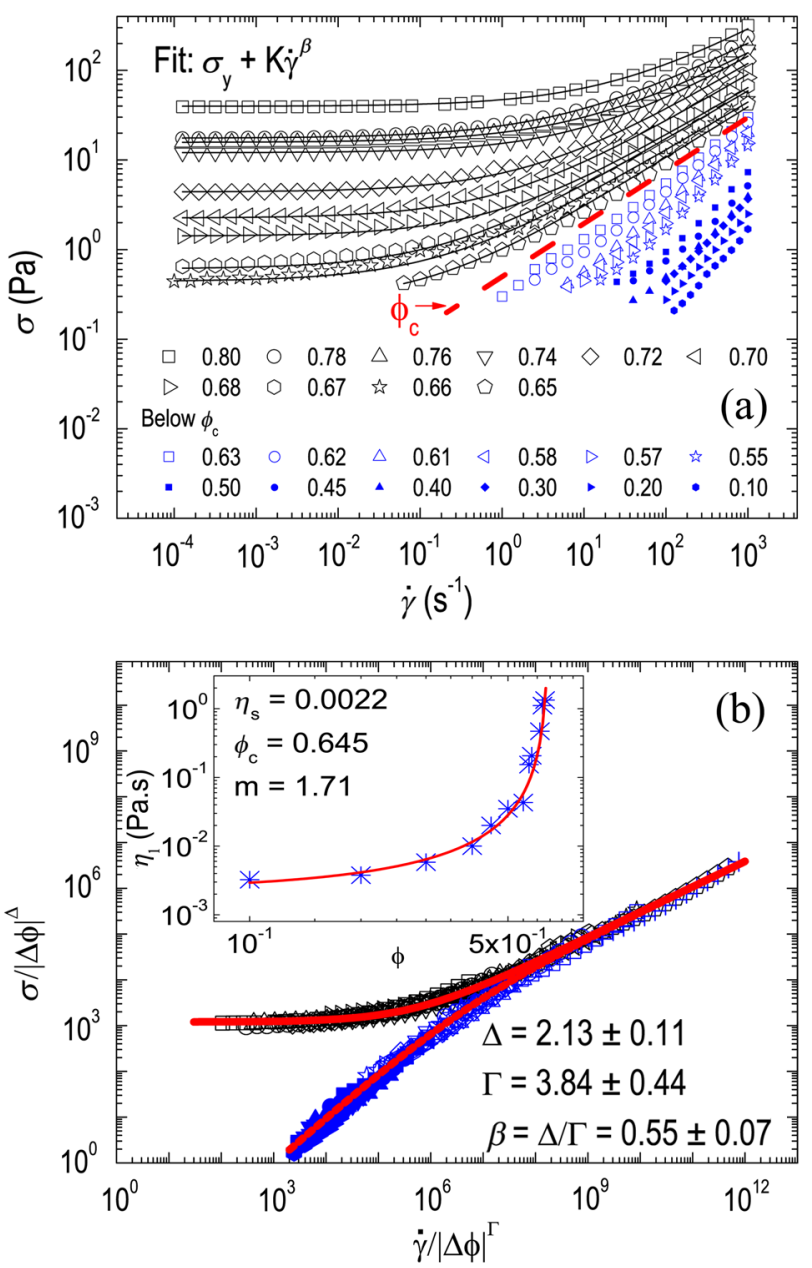

FIG. 1 (color online). (a) Flow curves of emulsions with different internal volume fractions, showing Herschel-Bulkley fittings for samples with $\phi>\phi_{c}$. (b) Master curve showing collapse of flow curves onto two branches, one for samples with $\phi>\phi_{c}$ and one for samples with $\phi<\phi_{c}$ when plotted as $\sigma /|\Delta \phi|^{\Delta}$ versus $\dot{\gamma} /|\Delta \phi|^{\Gamma}$; the red lines in the supercritical and the subcritical branches represents the Herschel-Bulkley and the Cross fit of the master curve, respectively, both with $\beta=0.55$ and $K=0.87$. Black symbols correspond to samples with $\phi>\phi_{c}$, and blue symbols correspond to samples with $\phi<\phi_{c}$. Inset: Fit of the low-shear viscosity to the Krieger-Dougherty equation, giving $\eta_{s}=2.2 \times 10^{-3} \mathrm{~Pa} \cdot \mathrm{s}$ and $m=1.71$ with $\phi_{c}=0.645$.

$\sigma^{\prime}=\eta_{s} \phi_{c}^{m} \dot{\gamma}^{\prime} /\left(1+\left(\eta_{s} \phi_{c}^{m} / K\right) \dot{\gamma}^{\prime 1-\beta}\right)$, where $\sigma^{\prime}$ and $\dot{\gamma}^{\prime}$ are the rescaled stress and shear rate, respectively. The fit of the master curve for $\phi<\phi_{c}$ is then given by the same parameters $\beta=0.55$ and $K=0.87$ as in the HerschelBulkley equation, giving an excellent fit with a viscosity $\eta_{s}=2.1 \times 10^{-3} \mathrm{~Pa} \cdot \mathrm{s}$ for the suspending medium, i.e., the water-SDS mixture. The rescaling of flow curves below $\phi_{c}$ also implies that for the low-shear regime, the KriegerDougherty equation can be written in the form $\sigma /\left|\Delta \phi^{\Delta}\right|=$ $\eta_{s} \phi_{c}^{m} \dot{\gamma} /|\Delta \phi|^{\Delta+m}$, whence $\Gamma=m+\Delta$. Therefore, the Krieger-Dougherty exponent $m$ can also be calculated only from the exponents $\Delta$ and $\Gamma(m=\Gamma-\Delta=1.71)$. An independent fit of the low-shear viscosities to 
the Krieger-Dougherty equation gives $m=1.71$ and $\eta_{s}=2.2 \times 10^{-3} \mathrm{~Pa} \cdot \mathrm{s}$, all in excellent agreement [Fig. 1(b), inset].

The scaling of the yield stress is interesting; however most models in this area focus on elastic moduli [18-20], notably the shear modulus $G^{\prime}$ since usually systems are incompressible. For very different systems $G^{\prime}$ is reported to vary as a power of the distance to jamming. An exponent $d \nu$ is suggested for dense repulsive gels [21]; with the spatial dimension $d=3$ and a correlation exponent $\nu \approx$ 0.7 [7] this would give an exponent around 2. However, from simulations with, e.g., harmonic or Hertzian interactions, different shear-modulus exponents are reported $[7,20]$. To quantify this for our system and see how it is related to the yield stress, we carry out oscillatory measurements using the CSS-MCR 300 Rheometer, which allows us to measure the storage $\left(G^{\prime}\right)$ and the loss $\left(G^{\prime \prime}\right)$ moduli for samples with $\phi>\phi_{c}$. We also measure both the stress and strain at which $G^{\prime}$ and $G^{\prime \prime}$ cross, which are measures for the yield stress and strain [Figs. 2(a) and 2(b)] [1]. This is because at high stresses and deformations the viscous contribution is observed to dominate the elastic one, indicating that the material is indeed flowing. Therefore, the crossover point defines both a yield stress and a yield strain $\left(\gamma_{y}\right)$. In Fig. 2(c) we show that yield stresses obtained by means of the Herschel-Bulkley equation (Fig. 1) and from the crossover of $G^{\prime}$ and $G^{\prime \prime}$ give very similar results, scaling as $|\Delta \phi|^{2.1}$. In the linear regime, the
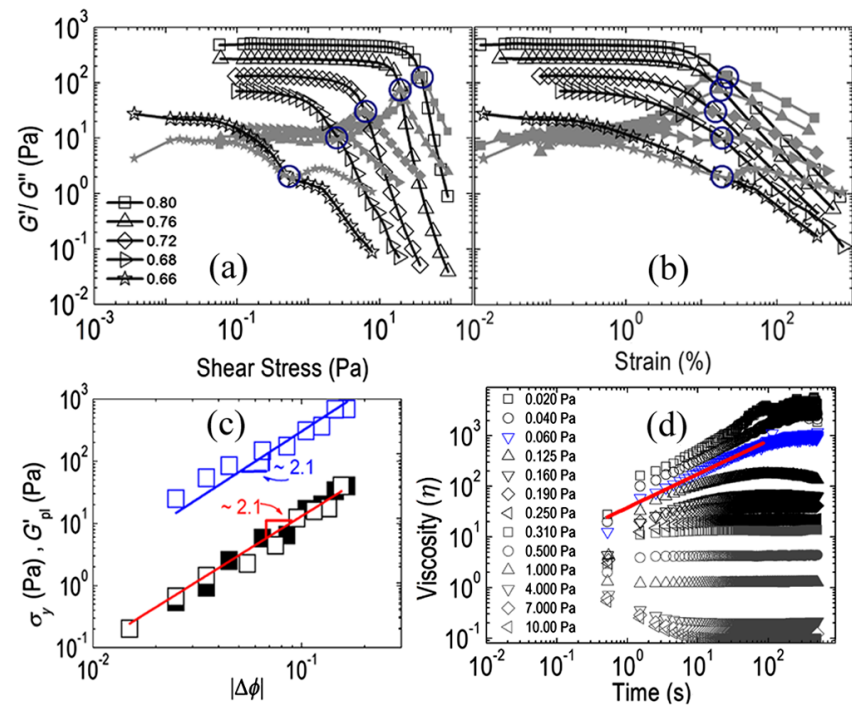

FIG. 2 (color online). Storage $\left(G^{\prime}\right)$ and loss $\left(G^{\prime \prime}\right)$ moduli as a function of the shear stress (a) and the strain (b). Black lines and symbols correspond to $G^{\prime}$, while gray lines and symbols correspond to $G^{\prime \prime}$; blue circles represent crossover points. (c) Scaling of the yield stress $\left(\sigma_{y}\right)$ with $|\Delta \phi|$, as determined from the Herschel-Bulkley equation (hollow black symbols) and from the crossover of $G^{\prime}$ and $G^{\prime \prime}$ (filled black symbols); scaling of $G_{\text {plateau }}^{\prime}$ with $|\Delta \phi|$ (blue symbols). (d) Behavior of a sample with $\phi=0.66$ of the viscosity $(\eta)$ in time under different imposed stresses; the red line indicates that $\eta \sim t^{\alpha}$, with $\alpha=0.6$. yield stress should obey the relation: $\sigma_{y}=G_{\text {plateau }}^{\prime} \gamma_{y}$ [22-24]. In Fig. 2(b) we observe that $\gamma_{y}$ is approximately constant for all samples $\left(\gamma_{y} \approx 0.2\right)$; therefore, $G_{\text {plateau }}^{\prime}$ should scale with $|\Delta \phi|$ the same way $\sigma_{y}$ does, which indeed is the case as also shown in Fig. 2(c). In addition, in agreement with the models quoted above, the exponent for the variation of $G^{\prime}$ with $|\Delta \phi|$ is indeed close to two.

Based on the experimentally obtained scalings, we now propose a simple model of heterogeneous dynamics. The observed phenomena are common to many experimental and simulated systems for which such dynamics has been reported (see, e.g., [25-29]). It has been suggested recently that the underlying cause is a generic critical transition in the dynamics, between a stagnant and a fluid phase (see, e.g., [27,28,30]). Here we follow this suggestion and attempt to explain the data from a simple and ad hoc two-state model for such a transition.

Since under stress the emulsions with $\phi>\phi_{c}$ start flowing, we assume that under stress only a fraction $s$ of the particles remains arrested in cages while the fraction $1-s$ becomes fluidized. The time-dependent heterogeneous pattern of stagnant and mobile regions is characterized by a length scale $\xi$ that increases when $s$ approaches a critical value $s_{c}$. In the spirit of critical transitions we assume $\xi$ to be the dominant variable, so $\xi \sim\left|1-s / s_{c}\right|^{-\nu}$, while other variables exhibit a power-law dependence on $\xi$. We assign a local viscosity $\eta_{f}$ to the fluidized phase and treat the arrested domains as a dispersed solid phase. Hence we postulate a viscosity $\eta=\eta_{f}\left(1-s / s_{c}\right)^{-m}$, with the same exponent $m$ as in the low-shear limit below $\phi_{c}$. The associated diverging time scale $\tau_{\eta}(s)$ is much shorter than the lifetime of the heterogeneity pattern, i.e., the time $\tau_{A}$ in which mobile particles get arrested again; hence $\tau_{A}(s) \sim\left(1-s / s_{c}\right)^{-n}$ with $n>m$. Following this, the competition between stress-induced relaxation out of the cage and fluctuation-induced arrest reads: $d s / d t=$ $-s / \tau_{R}+(1-s) / \tau_{A}(s)$. The relaxation rate $1 / \tau_{R}$ can be determined as the sum or maximum of either an Eyringtype rate for stress-induced escape out of a cage or the rate $\dot{\gamma}=\sigma / \eta$ at which the average cage vanishes. The Eyringtype rate has a very deep minimum at $\sigma=0$ and a steep increase towards a plateau value for increasing $\sigma$. The ratio $\tau_{A}(0) / \tau_{R}(0)$ is a very small number and is effectively zero above $\phi_{c}$; below $\phi_{c}$ it rapidly increases.

The full flow curve then follows from the steady-state solution $d s / d t=0$, which gives $s$ vs. $\sigma$ and subsequently the shape of the flow curve via $\eta(\sigma) \sim \tau_{A}(s)^{m / n}$. In particular the limiting cases when $s$ approaches $s_{c}$ from below and $s$ approaches 0 can be recognized directly from $\tau_{R} \gg$ $\tau_{A}(0)$ and $\tau_{R} \ll \tau_{A}(0)$, respectively. For $\sigma$ approaching 0 we then find $\eta / \eta_{f}=\left[\tau_{R}(0) / \tau_{A}(0)\right]^{m / n}$, implying an infinite viscosity and hence a yield stress above $\phi_{c}$. For $\sigma>\sigma_{y}$ the function $\tau_{R}$ equals $\dot{\gamma}^{-1}$, from which it is easy to derive the shear-thinning regime $\sigma=K \dot{\gamma}^{\beta}$ of the 
Herschel-Bulkley expression, with $K=\eta_{f} /\left[\tau_{A}(0)\right]^{m / n}$ and $\beta=1-m / n$; since $m=\Gamma-\Delta$ we can now make the identification $n \equiv \Gamma$. The yield stress itself follows selfconsistently from $1 / \tau_{R}(\sigma)=\dot{\gamma}$, with the result that the crossover rate to shear thinning decreases to effectively zero for decreasing $|\Delta \phi|$ and $\sigma_{y}$.

Below $\phi_{c}$ a very similar analysis holds as above, except that now the ratio $\tau_{R}(0) / \tau_{A}(0)$ and hence the low-stress viscosity is finite and rapidly decreases with decreasing $\phi$. Shear thinning now starts at $\dot{\gamma}=1 / \tau_{R}(0)$, which increases again with increasing $|\Delta \phi|$.

To understand the origin of the heterogeneity time scale and the associated exponent $n>m$, we make a variation on Mott's argument for explaining creep via dislocation motion in metals [31] and consider a collectively rearranging region of $N$ particles that share one collective free volume $v$ to make rearrangements possible. In a time $\tau_{\eta}$ the $N$ particles will on average each have moved to a neighboring position. Associated with this, each particle will experience an individual free-volume change $\delta v$, which will typically scale as $v / N$. The fluctuations $\delta v$ will be of either sign and if we assume them to be random, we have for the average over this time $\left\langle\sum \delta v\right\rangle=0,\left\langle\sum(\delta v)^{2}\right\rangle \sim$ $N(v / N)^{2}$. To have a finite probability that the free volume vanishes and the region is arrested we should have $\left\langle\Sigma(\delta v)^{2}\right\rangle \sim v^{2}$. To that end the process should be repeated $N$ times, whence $\tau_{A}=N \tau_{\eta}$. With $N \sim \xi^{d}$, we then find $n \equiv \Gamma=m+d \nu$ or $\Delta=d \nu$. Note that $\Delta$ is the yieldstress exponent and that we derived it here from the rheology, without considering the elastic interactions; when $\gamma_{y}$ is indeed constant (as it is in the experiments) $\Delta$ is then also the exponent of the shear modulus. So the model explains the experimentally observed yield-stress $\left(\phi>\phi_{c}\right)$ versus Newtonian-liquid behavior $\left(\phi<\phi_{c}\right)$ at low rate as well as the continuity across $\phi_{c}$ in the power-law shear-thinning regime at high rate, with microscopic interpretations for the scaling exponents.

So far, we have only considered the steady-state rheology. However, one of the key issues in the behavior of yield-stress fluids is the creep behavior. Over the past decades, there has been a discussion about whether the yield stress marks a transition between two liquidlike materials with very different viscosities, or whether the transition is truly between a liquid and something that behaves mechanically like a solid [32]. The distinction between the two is not a priori evident, since over long time scales a solid may creep. It was demonstrated recently [33] that the creep was the reason for the (wrongly interpreted) "liquidlike behavior" of yieldstress materials below $\sigma_{y}$; in fact the creep is so slow that a steady state is never achieved and so the apparent "viscosity" keeps on increasing in time.

From the model, a power-law creep behavior at stresses lower than $\sigma_{y}$ follows naturally. In the nonequilibrium regime where $s$ is already close to $s_{c}$ so that in the equation for $d s / d t$, approximately constant fractions $s_{c}$ and $1-s_{c}$ can be assumed and where $\sigma$ is so low that the rate of cage escape can still be neglected compared to the arrest rate, the rate equation can be integrated straightforwardly. The result is $\left(1-s / s_{c}\right) \sim$ $\left[t / \tau_{A}(0)\right]^{-1 /(n-1)}$, which directly gives the Andrade law $\eta \sim\left[t / \tau_{A}(0)\right]^{\alpha}$ with $\alpha=m /(n-1)$ [34]. Note that in this creep, with a rate $d s / d t$ that is independent of $\tau_{R}(\sigma)$, the system is far from equilibrium, so that $\alpha$ differs from the linear-response exponent $1-\beta=$ $m / n$. From the measured exponents in steady state, the model then predicts for the Andrade-creep exponent $m /(n-1) \equiv \alpha=0.60$. To test this prediction, we carry out creep measurements using a vane-in-cup geometry in the CSS-rheometer Stresstech: the shear stress is imposed and the resulting deformation in time is recorded. Due to the rheometer's resolution, the strain increase could be measured reliably only for samples with $\phi=0.66$ and $\phi=0.67$. Figure 2(d) shows that when a stress below $\sigma_{y}$ is imposed the material creeps and the apparent viscosity indeed increases as $\eta \sim t^{\alpha}$, with $\alpha \approx 0.6$, in line with the model and earlier observations on a range of different systems [33].

The model thus predicts both the fully steady-state Herschel-Bulkley and Cross regimes and the continuous transition between them. It makes use of only two independent exponents $m$ and $n$, which characterize the divergence of two time scales: one for the particle fluidity and one for the heterogeneity fluctuations. Similar time scales and their power-law behavior have been demonstrated in simulations of glasses [35]. The shear-thinning exponent $(0<\beta<1)$ is a direct consequence of the assumed exponent inequality $n>m$, for which a microscopic explanation is given here. Finally, the model describes the transient creep behavior under sub-critical stresses, giving a theoretical basis for the Andrade-creep equation and accurately reproducing its exponent value.

In conclusion, we demonstrated experimentally that by rescaling the shear stress and the shear rate with respect to $|\Delta \phi|$ it is possible to obtain master curves for the flow properties both above and below jamming, with a continuous transition between the two in the shear-thinning region. The curves above and below $\phi_{c}$ are not only accurately described by the Herschel-Bulkley and Cross equations, respectively, but we also show that the continuity across $\phi_{c}$ implies that the parameters are common between the two models. In addition, not only are the scaling exponents for stress and shear rate interconnected via the shear-thinning exponent, but the rescaling also predicts the KriegerDougherty exponent for the divergence of the viscosity when approaching $\phi_{c}$ from below. All this behavior and the Andrade creep can be captured by a simple and generic two-state model based on heterogeneous dynamics.

We thank Martin van Hecke and Brian Tighe for very helpful discussions. 
[1] R. G. Larson, The Structure and Rheology of Complex Fluids (Oxford University, New York, 1999).

[2] W. H. Herschel and R. Bulkley, Kolloid Z. 39, 291 (1926).

[3] M. Cross, J. Colloid Sci. 20, 417 (1965).

[4] I. M. Krieger and T. J. Dougherty, J. Rheol. 3, 137 (1959).

[5] G. Ovarlez, F. Bertrand, and S. Rodts, J. Rheol. 50, 259 (2006); N. Huang and D. Bonn, J. Fluid Mech. 590, 497 (2007).

[6] A. Liu and S.R. Nagel, Nature (London) 396, 21 (1998).

[7] C. S. O'Hern, L.E. Silbert, A. Liu, and S. Nagel, Phys. Rev. E 68, 011306 (2003).

[8] P. Olsson and S. Teitel, Phys. Rev. Lett. 99, 178001 (2007).

[9] M. Otsuki and H. Hayakawa, Phys. Rev. E 80, 011308 (2009).

[10] B. P. Tighe, E. Woldhuis, J. J. C. Remmers, W. van Saarloos, and M. van Hecke, Phys. Rev. Lett. 105, 088303 (2010).

[11] V. Trappe, V. Prasad, L. Cipelletti, P. N. Segre, and D. A. Weitz, Nature (London) 411, 772 (2001).

[12] K. N. Nordstrom, E. Verneuil, P.E. Arratia, A. Basu, Z. Zhang, A. G. Yodh, J.P. Gollub, and D. J. Durian, Phys. Rev. Lett. 105, 175701 (2010).

[13] A. Ikeda, L. Berthier, and P. Sollich, Phys. Rev. Lett. 109, 018301 (2012); arXiv:1302.4271v1.

[14] A. Fall, J. Paredes, and D. Bonn, Phys. Rev. Lett. 105, 225502 (2010).

[15] P. Møller, A. Fall, V. Chikkadi, D. Derks, and D. Bonn, Phil. Trans. R. Soc. A 367, 5139 (2009).

[16] L. Bécu, S. Manneville, and A. Colin, Phys. Rev. Lett. 96, 138302 (2006).
[17] V. Bertola, F. Bertrand, H. Tabuteau, D. Bonn, and P. Coussot, J. Rheol. 47, 1211 (2003).

[18] F. H. Stillinger and Z.W. Salsburg, J. Chem. Phys. 46, 3962 (1967).

[19] P.C.F. Møller and D. Bonn, Europhys. Lett. 80, 38002 (2007).

[20] B. P. Tighe, arXiv:1205.2960.

[21] X. Xing, S. Mukhopadhyay, and P. M. Goldbart, Phys. Rev. Lett. 93, 225701 (2004).

[22] M. M. Denn and D. Bonn, Rheol. Acta 50, 307 (2011).

[23] P. C. F. Møller, J. Mewis, and D. Bonn, Soft Matter 2, 274 (2006).

[24] H. A. Barnes and Q. D. Nguyen, J. Non-Newtonian Fluid Mech. 98, 1 (2001).

[25] R. A. Riggleman, H.-N. Lee, M. D. Ediger, and J. J. de Pablo, Soft Matter 6, 287 (2010).

[26] C. Heussinger, L. Berthier, and J.-L. Barrat, Europhys. Lett. 90, 20005 (2010).

[27] L. Laurson, M.-C. Miguel, and M. J. Alava, Phys. Rev. Lett. 105, 015501 (2010).

[28] J. Rosti, J. Koivisto, L. Laurson, and M. J. Alava, Phys. Rev. Lett. 105, 100601 (2010).

[29] S. Maccarrone et al., Soft Matter 6, 5514 (2010).

[30] M.-C. Miguel, L. Laurson, and M. J. Alava, Eur. Phys. J. B 64, 443 (2008).

[31] N. F. Mott, Philos. Mag. 44, 742 (1953).

[32] H. A. Barnes and K. Walters, Rheol. Acta 24, 323 (1985).

[33] P. C. F. Møller, A. Fall, and D. Bonn, Europhys. Lett. 87, 38004 (2009).

[34] E. N. da C. Andrade, Proc. R. Soc. A 84, 1 (1910).

[35] K. Kim and S. Saito, Phys. Rev. E 79, 060501(R) (2009); J. Chem. Phys. 138, 12 A506 (2013). 